\title{
On relative integral bases for unramified extensions
}

\author{
by \\ Kevin Hutchinson (Dublin)
}

0. Introduction. Since $\mathbb{Z}$ is a principal ideal domain, every finitely generated torsion-free $\mathbb{Z}$-module has a finite $\mathbb{Z}$-basis; in particular, any fractional ideal in a number field has an "integral basis". However, if $K$ is an arbitrary number field the ring of integers, $A$, of $K$ is a Dedekind domain but not necessarily a principal ideal domain. If $L / K$ is a finite extension of number fields, then the fractional ideals of $L$ are finitely generated and torsion-free (or, equivalently, finitely generated and projective) as $A$-modules, but not necessarily free. Beginning with some classical results of Artin and Chevalley (Propositions 1.1 and 1.2), we give some criteria for the existence or nonexistence of $A$-bases for ideals in $L$ or for the ring of integers of $L$ in the case where $L / K$ is unramified (Theorem 1.10 and Corollary 2.3). In particular, we show how the existence of an integral basis is (under mild hypotheses) determined by purely group-theoretic properties of the Galois group of the normal closure of $L / K$. We prove the main results for arbitrary finite separable field extensions $L / K$. The arguments were suggested by reading $[4]$.

1. Unramified extensions. We begin by recalling some of the basic facts about lattices (finitely generated torsion-free modules) over a Dedekind domain. If $P$ is a lattice over the Dedekind domain $A$, then $P \cong I_{1} \oplus \ldots \oplus I_{n}$ where $I_{1}, \ldots, I_{n}$ are ideals of $A$ and furthermore $I_{1} \oplus \ldots \oplus I_{n} \cong J_{1} \oplus \ldots \oplus J_{m}$ if and only if $n=m$ and $I_{1} \ldots I_{n} \cong J_{1} \ldots J_{m}$. Note also that if $I$ and $J$ are fractional ideals of $A$, then $I \cong J$ if and only if $[I]=[J]$, where $[K]$ denotes the class of the ideal $K$ in $C l(A)$, the ideal classgroup of $A$. It follows that the module $P \cong I_{1} \oplus \ldots \oplus I_{n}$ is determined up to isomorphism by its rank, $n$, and the class $\left[I_{1} \ldots I_{n}\right] \in C l(A)$, called the Steinitz class of $P$ and denoted $c(P)$. For example, if $J \subseteq A$ is an ideal representing $c(P)$ then $P \cong A^{\oplus(n-1)} \oplus J$. In particular $P$ has an $A$-basis (i.e., $P$ is free as an $A$-module) if and only if $c(P)=1$. (For details, see for example [1], [3] or [5].)

Suppose now that $A$ is a Dedekind domain with field of fractions $K$ and 
that $L / K$ is a finite separable extension of fields of degree $n$. Let $B$ be the integral closure of $A$ in $L$. Then $B$ is a Dedekind domain and any fractional ideal $I$ of $B$ is an $A$-lattice of rank $n$. We recall the following basic results on the Steinitz class of such a lattice:

Proposition 1.1. If I is any fractional ideal of $B$ then

$$
c(I)=c(B) \mathrm{N}_{L / K}[I] .
$$

Proposition 1.2. If $\delta_{B / A}$ is the relative discriminant of $B$ over $A$ and if $d_{L / K}$ is the discriminant of any $K$-basis of $L$, then

$$
\delta_{B / A}=J^{2}\left(d_{L / K}\right)
$$

where $J$ is a fractional ideal of $A$ representing the ideal class $c(B)$.

(For proofs, see [3].)

Here are some simple corollaries:

COROLlary 1.3. There exists an ideal of $B$ which has an $A$-basis if and only if

$$
c(B) \in \mathrm{N}_{L / K}(C l(B)) .
$$

Proof. By $1.1, I$ is $A$-free $\Leftrightarrow 1=c(I)=\mathrm{N}_{L / K}[I] c(B) \Leftrightarrow c(B)=$ $\mathrm{N}_{L / K}\left[I^{-1}\right]$.

COROllary 1.4.

$$
c(B)^{2}=\left[\delta_{B / A}\right]=\mathrm{N}_{L / K}\left[D_{B / A}\right]
$$

where $D_{B / A}$ is the different of $B$ relative to $A$.

Proof. This is immediate from 1.2.

Corollary 1.5. If $n$ is odd, there exists an ideal of $B$ which has an A-basis.

More generally, if the torsion abelian group $C l(A) / \mathrm{N}_{L / K} C l(B)$ has no nontrivial 2-torsion there exists a fractional ideal of $B$ with an $A$-basis.

Pr o of. Since $\left[c(B) \mathrm{N}_{L / K} C l(B)\right]^{2}=1$ in $C l(A) / \mathrm{N}_{L / K} C l(B)$, by 1.4 , the hypothesis implies that $c(B) \in \mathrm{N}_{L / K} C l(B)$ and hence there exists an $A$-free fractional ideal of $B$.

We will give an explicit example below of an extension of number fields $L / K$ where no fractional ideal of $L$ has a basis over the ring of integers of $K$ (Example 1.14).

Recall that if no prime of $A$ ramifies in $B$, then $\delta_{B / A}=A$.

Corollary 1.6. If no prime of $A$ ramifies in $B$ and if $C l(A)$ has no nontrivial 2-torsion, then $B$ has an A-basis.

Pr o of. Since $\delta_{B / A}=A$, we have $c(B)^{2}=\left[\delta_{B / A}\right]=1$ by 1.4 and hence $c(B)=1$ by hypothesis. 
If $D$ is a Dedekind domain, let $U(D)$ denote the group of units of $D$. Thus we have:

Corollary 1.7. Suppose that no prime of $A$ ramifies in $B$ and that $d_{L / K}$ is the discriminant of any K-basis of $L$. Then $B$ has an $A$-basis if and only if $d_{L / K}=u a^{2}$ with $u \in U(A)$ and $a \in K^{*}$.

Proof. By 1.2, $A=J^{2}\left(d_{L / K}\right)$ where $J$ represents $c(B)$. Thus, $\left(d_{L / K}\right)=$ $J^{-2}$ and hence $B$ is $A$-free $\Leftrightarrow J$ is a principal ideal $\Leftrightarrow\left(d_{L / K}\right)$ is the square of a principal ideal $\Leftrightarrow d_{L / K}=u a^{2}$.

Suppose now that $\theta$ is a primitive element for $L / K$. Let $E$ be the normal closure of $L / K$ and let $G$ be the Galois group of $E / K, H$ the Galois group of $E / L$. Let $\left\{\sigma_{1}, \ldots, \sigma_{n}\right\}$ be a set of representatives for the elements of the coset space $G / H$. Let $d=d(\theta)=d_{L / K}\left(1, \theta, \ldots, \theta^{n-1}\right)=\prod_{i \neq j}\left(\sigma_{i}(\theta)-\sigma_{j}(\theta)\right)=$ $\alpha(\theta)^{2}$ where $\alpha=\alpha(\theta)=\prod_{i<j}\left(\sigma_{i}(\theta)-\sigma_{j}(\theta)\right)$. Finally, let $C$ be the integral closure of $A$ in $E$.

Lemma 1.8. If no prime of $A$ ramifies in $B$ and if either $U(C)^{2} \cap K=$ $U(A)^{2}$ or $[E: L]$ is odd and $U(B)^{2} \cap K=U(A)^{2}$, then $B$ has an $A$-basis if and only if $\alpha \in K$.

Pr o o f. If $\alpha \in K$ then $d=\alpha^{2}$ in $K$ and hence $B$ is $A$-free by 1.7 (without the added hypotheses on squares of units). Conversely, suppose that $B$ is $A$ free. Then $\alpha^{2}=d=u a^{2} \Rightarrow\left(a^{-1} \alpha\right)^{2}=u \Rightarrow u \in U(C)^{2} \cap K \Rightarrow u \in U(A)^{2} \Rightarrow$ $\alpha^{2}=(v a)^{2}$ for some $v \in U(A) \Rightarrow \alpha= \pm v a \in K$ if $U(C)^{2} \cap K=U(A)^{2}$. If $[E: L]$ is odd then $\alpha \in L$ and thus in the argument just given, $a^{-1} \alpha \in L$ and hence $u \in U(B)^{2} \cap K$.

Note. The condition on units $U(B)^{2} \cap K=U(A)^{2}$ is not very restrictive. In the number field case, for instance, there are only finitely many quadratic extensions of the field $K$ of the form $K(\sqrt{u}) / K$ where $u$ is a unit of $K$ and the condition simply says that any such extension is not contained in $L$.

Recall that if $\sigma$ is a permutation of the set $\left\{x_{1}, \ldots, x_{n}\right\}$, then $\sigma$ is an even permutation if and only if

$$
\sigma\left(\prod_{i<j}\left(x_{i}-x_{j}\right)\right)=\prod_{i<j}\left(x_{i}-x_{j}\right)
$$

Thus $\alpha(\theta) \in K \Leftrightarrow \sigma(\alpha(\theta))=\alpha(\theta)$ for all $\sigma \in G \Leftrightarrow \sigma$ acts as an even permutation on $\left\{\sigma_{1}(\theta), \ldots, \sigma_{n}(\theta)\right\}$ for all $\sigma \in G \Leftrightarrow$ each $\sigma \in G$ acts evenly on the $G$-set $G / H$ since the map $G / H \rightarrow\left\{\sigma_{1}(\theta), \ldots, \sigma_{n}(\theta)\right\}, \sigma_{i} H \mapsto \sigma_{i}(\theta)$ is an isomorphism of $G$-sets.

We will say that the group $G$ acts evenly on the $G$-set $X$ if each element of $G$ acts on $X$ as an even permutation. Otherwise we will say that $G$ acts oddly on $X$. 
Lemma 1.9. Let $G$ be a finite group and $H$ a subgroup of odd order. Then $G$ acts oddly on $G / H$ if and only if the Sylow 2-subgroups of $G$ are nontrivial and cyclic.

P r o of. Since every element of odd order in a permutation group is even, $G$ acts oddly on a set $X$ if and only if some element of $G$ of 2-power order acts oddly. Suppose that $\sigma \in G, \sigma \neq 1$ has 2-power order and let $C$ be the cyclic subgroup of $G$ generated by $\sigma$. Let $\tau \in G$ and consider the orbit of $\tau H \in G / H$ under $C$. The stabilizer of $C$ on $\tau H$ is $C \cap \tau H \tau^{-1}=1$ since $\tau H \tau^{-1}$ has odd order and $C$ has 2-power order. Thus $G / H$ decomposes into $[G: H] /|C|$ orbits each of length $|C|$. Thus, as a permutation, $\sigma$ factors as a product of $[G: H] /|C|$ cycles, each of length $|C|$. But each cycle of length $|C|$ in turn factors as a product of $|C|-1$ transpositions and hence $\sigma$ factors as a product of $\frac{[G: H]}{|C|}(|C|-1)$ transpositions. Since $|C|-1$ is odd, $\sigma$ acts oddly $\Leftrightarrow[G: H] /|C|$ is odd $\Leftrightarrow C$ is a Sylow 2-subgroup of $G$.

Combining 1.8 and 1.9 we obtain:

THeOREM 1.10. Suppose that $L / K$ is a finite separable extension of fields and that $E$ is the normal closure of $L / K$. Suppose that $A$ is a Dedekind domain with field of fractions $K$ and that $B$ and $C$ are the integral closures of $A$ in $L$ and $E$ respectively. If $[E: L]$ is odd and $U(B)^{2} \cap K=U(A)^{2}$ and if no prime of $A$ ramifies in $B$ then $B$ has an $A$-basis if and only if the Sylow 2-subgroup of $G$ is not nontrivial and cyclic.

This generalises the result (see [3]) that if $L / K$ is Galois, unramified of odd degree, then $B$ has an $A$-basis. However, here is an example of an unramified extension $L / K$ of odd degree for which $B$ is not free as an $A$ module.

EXAMPLE 1.11. Let $F$ be the splitting field of $f(X)=X^{3}-X+1$ over $\mathbb{Q}$. The discriminant of $f(X)$ is -23 , so $\operatorname{Gal}(F / \mathbb{Q})=S_{3}$, the symmetric group on three letters. Let $E=F(\sqrt{2})$ and $K=\mathbb{Q}(\sqrt{-46})$. $E$ is the splitting field of $f(X)$ over $\mathbb{Q}(\sqrt{2})$ and hence $E$ is unramified (at any finite prime) over $\mathbb{Q}(\sqrt{-23}, \sqrt{2})$ by the arguments of Uchida [6] (Theorem 1 and Corollary). $\mathbb{Q}(\sqrt{-23}, \sqrt{2})$ is in turn unramified over $K$ and thus $E / K$ is a Galois unramified extension with $\operatorname{Gal}(E / K)=S_{3}$. Let $H$ be any subgroup of $\operatorname{Gal}(E / K)$ of order 2 and let $L=E^{H}$. Let $A, B$ and $C$ be the rings of integers of $K, L$ and $E$ respectively. Since $U(A)=\{ \pm 1\}$ and $\sqrt{-1} \notin \mathbb{Q}(\sqrt{-23}, \sqrt{2})$ it follows that $U(C)^{2} \cap K=U(A)^{2}$. Since $S_{3}$ acts oddly on $S_{3} / H, \alpha \notin K$ and thus $B$ is not a free $A$-module by 1.8 .

If $[E: L]=|H|$ is even, then 1.9 is easily seen to fail and there is no simple criterion for $G$ to act oddly on $G / H$. However, in certain circumstances one can provide criteria. We will consider this below. 
For the present we specialize to the case where $L / K$ is an extension of number fields and $A$ is the ring of integers of $L$. In this situation classfield theory allows us to control the norm map $\mathrm{N}_{L / K}: C l(B) \rightarrow C l(A)$ :

Lemma 1.12. Let $K_{1}$ be the Hilbert classfield of $K$. Let $\varrho_{K}: C l(A) \rightarrow$ $\operatorname{Gal}\left(K_{1} / K\right)$ be the Artin isomorphism. Then $\varrho_{K}$ induces an isomorphism $\mathrm{N}_{L / K}(C l(B)) \rightarrow \operatorname{Gal}\left(K_{1} / K_{1} \cap L\right)$.

Proof. Let $L_{1}$ be the Hilbert classfield of $L$. Then $L_{1} \supseteq K_{1}$ and if $\varrho_{L}: C l(B) \rightarrow \operatorname{Gal}\left(L_{1} / L\right)$ is the Artin isomorphism for $L$ and $\operatorname{res}_{L / K}$ is the restriction map $\operatorname{Gal}\left(L_{1} / L\right) \rightarrow \operatorname{Gal}\left(K_{1} / K\right)$, then $\varrho_{K} \mathrm{~N}_{L / K}=\operatorname{res}_{L / K} \varrho_{L}$ and hence $\varrho_{K}$ induces an isomorphism $\mathrm{N}_{L / K}(C l(B)) \rightarrow \operatorname{res}_{L / K}\left(\operatorname{Gal}\left(L_{1} / L\right)\right)=$ $\operatorname{Gal}\left(K_{1} / L \cap K_{1}\right)$.

COROLlary 1.13. Suppose that $L / K$ is unramified and that $L$ contains the maximal abelian unramified 2-extension of $K$. Then there exists an ideal of $B$ with an $A$-basis if and only if $B$ has an $A$-basis.

Pro of. $L / K$ unramified $\Rightarrow c(B)^{2}=1$ and since $L$ contains the maximal abelian unramified 2-extension of $K, \mathrm{~N}_{L / K}(C l(B))$ has odd order by 1.12 . Thus $c(B)=1 \Leftrightarrow c(B) \in \mathrm{N}_{L / K}(C l(B))$.

ExAmple 1.14. Let $K=\mathbb{Q}(\sqrt{-14}), F=K(\sqrt{2}), L=K(\sqrt{2 \sqrt{2}-1})$. Then $L$ is the Hilbert classfield of $K$ (see, for example, Cox [2]). Clearly $\operatorname{Gal}(L / K) \cong C l(A)$ is cyclic of order 4 and $\operatorname{Gal}(F / K)$ is cyclic of order 2 . Let $B$ be the ring of integers of $L$ and let $C$ be the ring of integers of $F$. Note that $U(A)=\{ \pm 1\}$ and that $\sqrt{-1} \notin L$ (for otherwise we would have $\sqrt{-1} \in F=\mathbb{Q}(\sqrt{-14}, \sqrt{2})$ which is clearly false). It follows that $U(B)^{2} \cap K=$ $U(C)^{2} \cap K=U(A)^{2}=1$. Thus neither $B$ nor $C$ has an $A$-basis by 1.9 . No ideal of $B$ is $A$-free by 1.13 .

However $\mathrm{N}_{F / K}(C l(C))$ is the unique subgroup of order 2 in $C l(A)$ by 1.12 and thus, since $c(C)^{2}=1$ (because $F / K$ is unramified), $c(C) \in \mathrm{N}_{F / K}(C l(C)$ ) and so there exist ideals of $C$ which are $A$-free.

2. "Odd" group actions. In this section we prove a few results on oddness of transitive group actions where the stabilizer has even order. In the case where the stabilizer has a normal complement, a criterion for oddness can be given:

TheOREM 2.1. Suppose that $G$ is a finite group with subgroup H. Suppose that $H$ has a normal complement $N$. Let $S$ be a Sylow 2-subgroup of $H$ and suppose the elements $\sigma_{1}, \ldots, \sigma_{r}$, of orders $2^{m_{1}}, \ldots, 2^{m_{r}}$, generate $S$. Then $G$ acts oddly on $G / H$ if and only if either the Sylow 2-subgroups of $N$ are 
nontrivial and cyclic or

$$
\sum_{k=0}^{m_{i}-1} 2^{m_{i}-k-1}\left|C_{N}\left(\sigma_{i}^{2^{k}}\right)\right| \not \equiv\left(2^{m_{i}}-1\right)|N| \bmod 2^{m_{i}+1}
$$

for some $i \in\{1, \ldots, r\}$ where $C_{N}(\tau)=\{\mu \in N \mid \mu \tau=\tau \mu\}$ for $\tau \in G$.

Proof. Since $G=H N$ and since a product of two even permutations is even, $G$ acts oddly on $G / H$ if and only if either $H$ or $N$ acts oddly on $G / H$. Now, the bijection of sets $N \leftrightarrow G / H$ induces an isomorphism of $N$-sets if $N$ acts on $N$ by left multiplication and a bijection of $H$-sets if $H$ acts on $N$ by conjugation. Thus $N$ acts oddly on $G / H$ if and only if the Sylow 2-subgroup of $N$ is nontrivial and cyclic by Lemma 1.9 (with $G=N$ and $H=1$ ). Clearly $H$ acts oddly on $N$ by conjugation if and only if $S$ does. $S$ acts oddly on $N$ if and only if some $\sigma_{i}$ does. It remains to show that $\sigma_{i}$ acts as an odd permutation if and only if

$$
\sum_{k=0}^{m_{i}-1} 2^{m_{i}-k-1}\left|C_{N}\left(\sigma_{i}^{2^{k}}\right)\right| \not \equiv\left(2^{m_{i}}-1\right)|N| \bmod 2^{m_{i}+1} .
$$

Fix $i$ and let $\sigma=\sigma_{i}, m=m_{i}$. Let $r_{k}=\left|C_{N}\left(\sigma^{2^{k}}\right)\right|$. Consider the action of $\sigma$ on $N$ by conjugation. $N$ decomposes as a union of orbits of length $2^{k}$, $k \leq m$. If $\tau \in N$, then the orbit of $\tau$ has length $2^{k}$ if and only if $\sigma^{2^{k}}$ fixes $\tau$ but $\sigma^{2^{k-1}}$ does not; i.e., if and only if $\tau \in C_{N}\left(\sigma^{2^{k}}\right)-C_{N}\left(\sigma^{2^{k-1}}\right)$. Thus the number of orbits of length $2^{k}$ is

$$
s_{k}=\frac{1}{2^{k}}\left(r_{k}-r_{k-1}\right)
$$

Thus the permutation $\sigma$ factors as a product of the form

$$
\prod_{k=1}^{m}\left(\prod_{j=1}^{s_{k}} \sigma_{k j}\right)
$$

where $\sigma_{k j}$ is a $2^{k}$-cycle. Hence $\sigma_{k j}$ in turn factors as a product of $2^{k}-1$ transpositions and hence $\sigma$ factors as a product of $t$ transpositions where

$$
\begin{aligned}
t & =\sum_{k=1}^{m}\left(2^{k}-1\right) s_{k}=\sum_{k=1}^{m}\left(2^{k}-1\right) \frac{1}{2^{k}}\left(r_{k}-r_{k-1}\right) \\
& =\frac{1}{2^{m}} \sum_{k=1}^{m}\left(2^{m}-2^{m-k}\right)\left(r_{k}-r_{k-1}\right) \\
& =\frac{1}{2^{m}}\left\{2^{m}\left(r_{m}-r_{0}\right)-\sum_{k=1}^{m} 2^{m-k}\left(r_{k}-r_{k-1}\right)\right\} \\
& =\frac{1}{2^{m}}\left\{\left(2^{m}-1\right) r_{m}-2^{m-1} r_{0}-2^{m-2} r_{1}-\ldots-r_{m-1}\right\} .
\end{aligned}
$$


Thus $\sigma$ acts oddly $\Leftrightarrow t \not \equiv 0 \bmod 2 \Leftrightarrow 2^{m} t \not \equiv 0 \bmod 2^{m+1} \Leftrightarrow$

$$
\sum_{k=0}^{m-1} 2^{m-k-1} r_{k} \not \equiv\left(2^{m}-1\right) r_{m} \bmod 2^{m+1}
$$

proving the result since $r_{m}=\left|C_{N}\left(\sigma^{2^{m}}\right)\right|=\left|C_{N}(1)\right|=|N|$.

Corollary 2.2. Suppose $G$ is a Frobenius group with kernel $N$ and complement $H$. If $|H|$ is odd, then $G$ acts evenly on $G / H$. If $|H|$ is even, then $G$ acts oddly on $G / H$ if and only if the Sylow 2-subgroups of $H$ are cyclic of order $2^{m}$ and $2^{m+1}$ does not divide $|N|-1$.

Proof. Since it can easily be shown that the Sylow 2-subgroups of $N$ cannot be nontrivial cyclic, it follows that if $H$ has odd order then $G$ acts evenly on $G / H$ by 1.8. Suppose, on the other hand, that $H$ has even order. If $\sigma \in H-\{1\}$ then $C_{N}(\sigma)=1$. Suppose $\sigma \in H$ of order $2^{m}$. Then $\left|C_{N}\left(\sigma^{2^{k}}\right)\right|=$ 1 for $k \leq m-1$. Thus, by the proof of Theorem 2.1, $\sigma$ acts oddly on $G / H \Leftrightarrow$

$$
2^{m}-1 \not \equiv\left(2^{m}-1\right)|N| \bmod 2^{m+1}
$$

$\Leftrightarrow 2^{m+1}$ does not divide $|N|-1$. However, if $\sigma$ does not generate a Sylow 2-subgroup of $H$ then the order of such a group is $2^{k}$ with $k \geq m+1$ and hence $\sigma$ acts evenly since $2^{k}$ divides $|N|-1$ (because $|H|$ does). This proves the result.

A special case of 2.2 is the case where $G$ is dihedral of order $2 m$ with $m$ odd and $H$ is a subgroup of order 2 . Then $G$ acts oddly on $G / H$ if and only if $m \not \equiv 1 \bmod 4$.

Corollary 2.3. Suppose $E / K$ is a Galois extension of fields with $\operatorname{Gal}(E / K)=G$ a Frobenius group with complement $H$. Let $L$ be the fixed field of $H$. Suppose that $A$ is a Dedekind domain with field of fractions $K$ and that $B$ and $C$ are the integral closures of $A$ in $L$ and $E$ respectively. Suppose that no prime of $A$ ramifies in $B$ and that $U(C)^{2} \cap K=U(A)^{2}$. Then $B$ has an $A$-basis if and only if one of the following holds: (i) $|H|$ is odd or (ii) the Sylow 2-subgroup of $H$ is not cyclic or (iii) the Sylow 2-subgroup of $H$ is cyclic of order $2^{m}$ and $2^{m+1}$ divides $[L: K]-1$.

Proof. This follows at once from 2.2 and 1.8.

Of course we could have stated a more general result using Theorem 2.1 rather than 2.2 .

\section{References}

[1] E. Artin, Questions de base minimale dans la théorie des nombres algébriques, CNRS XXIV (Colloq. Int., Paris, 1949), 19-20. 
[2] D. A. Cox, Primes of the Form $x^{2}+n y^{2}$, Wiley, 1989.

[3] A. Fröhlich, Ideals in an extension field as modules over the algebraic integers in a finite number field, Math. Z. 74 (1960), 29-38.

[4] L. McCulloh, Frobenius groups and integral bases, J. Reine Angew. Math. 248 (1971), 123-126.

[5] E. Steinitz, Rechteckige Systeme und Moduln in algebraischen Zahlkörpern I, II, Math. Ann. 71 (1911), 328-353; 72 (1911), 297-345.

[6] K. Uchida, Unramified extensions of quadratic number fields I, Tôhoku Math. J. 22 (1970), 138-141.

DEPARTMENT OF MATHEMATICS

UNIVERSITY COLLEGE DUBLIN

BELFIELD, DUBLIN 4, IRELAND

E-mail: KHUTCH@IRLEARN.UCD.IE 